\title{
miR-202 acts as a potential tumor suppressor in breast cancer
}

\author{
SHANSHAN GAO ${ }^{1,2}$, CHUNFANG CAO ${ }^{2}$, QINGFU DAI ${ }^{2}$, JIAN CHEN $^{2}$ and JIANCHENG TU \\ ${ }^{1}$ Department of Laboratory Medicine, Clinical Laboratory Medicine and Center for Gene Diagnosis, \\ Zhongnan Hospital of Wuhan University, Wuhan, Hubei 430071; ${ }^{2}$ Central Laboratory, \\ Longyan First Hospital Affiliated to Fujian Medical University, Longyan, Fujian 364000, P.R. China
}

Received November 20, 2017; Accepted April 26, 2018

DOI: $10.3892 / \mathrm{ol} .2018 .8726$

\begin{abstract}
Breast cancer affects $\sim 10 \%$ of women worldwide and is responsible for $\sim 12 \%$ of all cancer-associated mortalities. Breast cancer is more prone to metastasis compared with other types of cancer. Up to $5 \%$ of patients with breast cancer present with incurable metastasis and an additional $10-15 \%$ of patients develop metastases within 3 years of their initial diagnosis. MicroRNAs (miRNAs) are short RNAs, 21-25 nucleotides in length, that have been shown to significantly affect gene expression. In total $>2,000$ miRNAs have been identified and specific miRNAs have been revealed to be associated with cancer. In the present study, we observed that the majority of breast cancer specimens collected expressed low levels of miR-202 compared with adjacent tissues and normal cell lines. Mechanistic investigations identified KRAS as a potential target gene of miR-202 and it was demonstrated that miR-202 exerted its tumor-suppressive effects by regulating the expression of KRAS in breast cancer cells. Functional assays revealed that miR-202 significantly reduced cell proliferation, migration and invasion in vitro. In summary, these results indicate the function of miR-202 in breast cancer progression and suggest that its use within breast cancer therapy is promising.
\end{abstract}

\section{Introduction}

Breast cancer is one of the most common malignant tumors in women. In many areas, breast cancer is the most common malignancy in females. In China, the incidence of breast cancer is increasing annually. Because the development and progression of breast cancer are complex processes involving many genes, the underlying mechanism of breast cancer remains unclear $(1,2)$.

Correspondence to: Mr. Jiancheng Tu, Department of Laboratory Medicine, Clinical Laboratory Medicine and Center for Gene Diagnosis, Zhongnan Hospital of Wuhan University, 169 Donghu Road, Wuhan, Hubei 430071, P.R. China

E-mail: jianchengtu@whu.edu.cn

Key words: breast cancer, KRAS, metastasis, miR-202, proliferation
Single-stranded RNAs consisting of 19 to 25 nucleotides are known as microRNAs (miRNAs). These RNAs are regulatory molecules that modulate the expression of functional genes, play an important role in many biological processes, and are expressed in a tissue- and time-specific manner. miRNAs regulate gene expression at the post-transcriptional level mainly via completely complementary or partially complementary binding to the 3' UTR of the target gene, resulting in degradation or translational repression of the target gene (2-4). Studies have shown that a variety of miRNAs are involved in malignant transformation processes, such as malignant proliferation, metastasis and recurrence, and apoptosis inhibition. Studies have reported that miRNA inhibitors can reduce the high miR-21 expression in breast cancer cells, inhibiting proliferation and migration; that miR-373 can promote the metastasis of breast cancer cells; that miR-520 plays a role in promoting the development of breast cancer; and that miR-126 and miR-335 are able to inhibit breast cancer to a certain extent (5-7). Recent studies have demonstrated that miR-202 is associated with several types of cancer, miR-202 has a lower expression in several of cancers, and however, the expression and function of miR-202 have not been investigated in breast cancer (8).

KRAS belongs to the RAS family; it is located on the short arm of chromosome 12 and is $35 \mathrm{~kb}$ long. KRAS is a downstream molecule of the EGF receptor. It possesses four exons and one 5' end non-coding exon, encoding a 189-aa RAS protein. This gene mainly functions in signaling pathways involving membrane receptors and cAMP. KRAS has the strongest influence on malignant cancer in the RAS family (9-12).

In our study, we found abnormal expression of miR-202 and KRAS in breast cancer. We also found that miR-202 may affect the proliferation and metastasis of breast cancer cells by regulating KRAS. Our results provide a new target for the diagnosis and treatment of breast cancer.

\section{Materials and methods}

Samples. Samples were collected from 30 patients (women aged 49-68 years) from May 2011 to 2013 at Zhongnan Hospital of Wuhan University (Wuhan, China). All patients reviewed the methods and significance of the study and provided written informed consent. The project was approved by the ethics committee for medical science research of Zhongnan Hospital 
Table I. Primer sequences.

\begin{tabular}{lll}
\hline Name & \multicolumn{1}{c}{ Forward primer (5'-3') } & \multicolumn{1}{c}{ Reverse primer (5'-3') } \\
\hline KRAS & TACCAFCAACTCCAACGG & GAACCCAAGGCATCTCCA \\
GAPDH & AGAAGGCTGGGGCTCATTTG & AGGGGCCATCCACAGTCTTC \\
microRNA-202 & CTCCAGAGAGAUAGUAGAGCCT & CTCAACCAATCACCTGGCACAGA \\
U6 & CTCGCTTCGGCAGCACA & AACGCTTCACGAATTTGCGT \\
\hline
\end{tabular}

of Wuhan University. All cancer tissues were pathologically confirmed to be breast cancer.

MicroRNA microarray analysis. A total of $500 \mathrm{ng}$ of RNA was subjected to Agilent miRNA microarray analysis (Guangzhou RiboBio Co., Ltd., Guangzhou, China). Differences between groups were examined for statistical significance with an unpaired Student's t test.

Cell culture. 293, MCF7 and '-MB-231 cells were cultured in DMEM supplemented with 10\% FCS (both Invitrogen; Thermo Fisher Scientific Inc., Waltham, MA, USA), 100 IU/ml penicillin and $100 \mathrm{mg} / \mathrm{ml}$ streptomycin (Shanghai Shenggong Biology Engineering Technology Service, Ltd., Shanghai, China) at $37^{\circ} \mathrm{C}$ in a humidified atmosphere containing $5 \% \mathrm{CO}_{2}$.

MTT assays. Cells were plated in 96-well plates at a density of $1 \times 10^{4}$ cells per well; $24 \mathrm{~h}$ later, the cells were transfected or treated as indicated. At the end of the incubation, cellular proliferation was measured by MTT assays. The optical density at $490 \mathrm{~nm}$ was measured using a microplate reader (Bio-Rad Laboratories, Inc., Hercules, CA, USA).

Metastasis assay. A total of $1 \times 10^{5}$ cells were seeded onto the upper part of a transwell chamber (BD Bioscience, Franklin Lakes, NJ, USA) containing a gelatin-coated polycarbonate membrane filter (pore size: $8 \mathrm{~mm}$ ) for migration and invasion assays. Serum-free medium was added to the upper well, and medium containing $10 \%$ FBS was added to the lower well. After $24 \mathrm{~h}$ of incubation at $37^{\circ} \mathrm{C}$ with $5 \% \mathrm{CO}_{2}$, the filters were stained with crystal violet (Amresco, LLC., Solon, OH, USA). Five random fields were counted per chamber by using an inverted microscope.

Real-time PCR. Total RNA was isolated with TRIzol (Invitrogen; Thermo Fisher Scientific Inc.) according to the manufacturer's protocol. Complementary DNA was synthesized by reverse transcription of total RNA using an RT reaction kit (Promega Corp., Madison, WI, USA). Real-time PCR was performed according to the manufacturer's instructions. SYBR Premix Ex Taq (TaKaRa Bio, Inc., Otsu, Japan) was used as a DNA-specific fluorescent dye. The primer sequences in Table I were synthesized.

All reactions were repeated at least three times. Gene expression levels were calculated relative to GAPDH or U6 with Stratagene Mx 3000P software.

Western blot analysis. Total proteins were extracted using radioimmunoprecipitation (RIPA) assay buffer (Wlaterson,
Table II. Differentially expressed miRNAs in breast cancer.

\begin{tabular}{lcl}
\hline miRNA & Fold change & Trend \\
\hline miR-202 & 38.92 & Down \\
miR-34 & 11.02 & Down \\
miR-29 & 8.43 & Down \\
miR-342 & 2.98 & Down \\
miR-21 & 17.98 & Up \\
miR-269 & 12.20 & Up \\
\hline
\end{tabular}

miR, microRNA.

Barcelona, Spain) and were quantified using a bicinchoninic acid (BCA) protein assay kit (Wlaterson). Equal amounts of protein $(60 \mu \mathrm{g})$ were boiled at $100^{\circ} \mathrm{C}$ for $5 \mathrm{~min}$, separated on $12 \%$ sodium dodecyl sulfate polyacrylamide gels (SDS-PAGE), and electrotransferred to polyvinylidene fluoride (PVDF) membranes (Millipore, Bedford, MA, USA). The membranes were blocked with $10 \%$ skim milk (w/v) at room temperature for $2 \mathrm{~h}$. Target proteins were probed with specific antibodies against KRAS and GAPDH (Santa Cruz, Biotechnology, Inc., Dallas, TX, USA). The membranes were stripped and re-probed with an antibody against GAPDH to verify equal loading.

Construction of stable siRNA-expressing cell lines. To stably silence KRAS, cells were transfected with the pRS-si-KRAS plasmid (Shanghai GeneChem Co., Ltd., Shanghai, China) and were selected with G418 $(400 \mu \mathrm{g} / \mathrm{ml})$. After 3 weeks, stable cells were selected, cultured and amplified.

Statistical analysis. All experiments were repeated at least three times. A log-rank test was used to evaluate the correlation between possible prognostic factors. Statistical significance between two groups of data was evaluated by Student's t test (two-tailed). One-way ANOVA and Dunnett's post hoc test were used for comparisons among multiple groups. Statistical analysis was performed using GraphPad Prism software (GraphPad Software, Inc., La Jolla, CA, USA). $\mathrm{P}<0.05$ was considered to indicate statistically significant difference.

\section{Results}

The expression of miR-202 and KRAS in breast cancer. Many miRNAs were abnormally expressed in breast cancer 
A
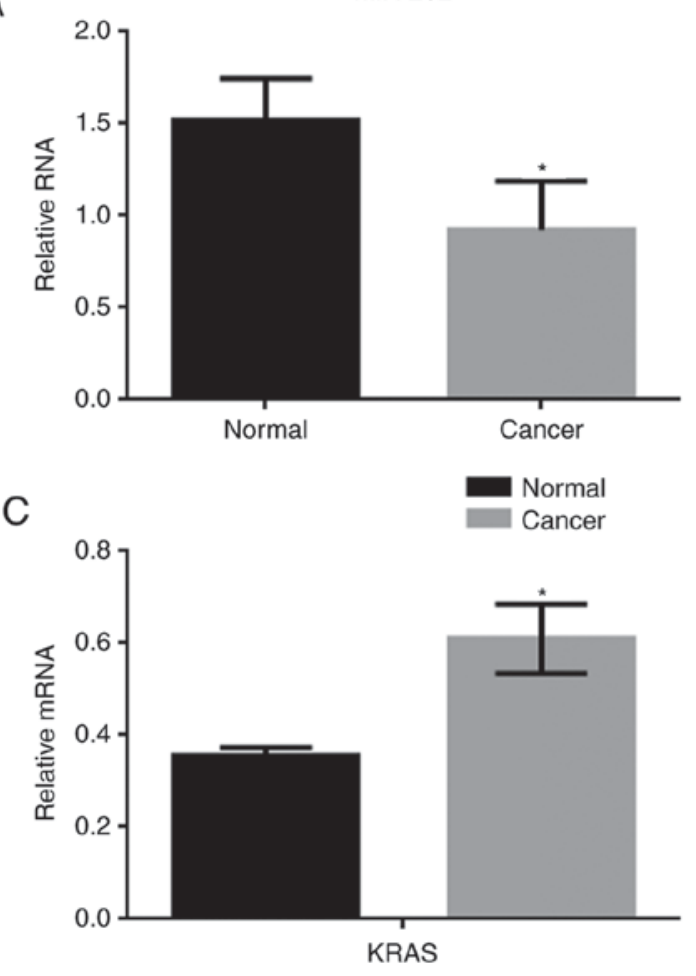

B

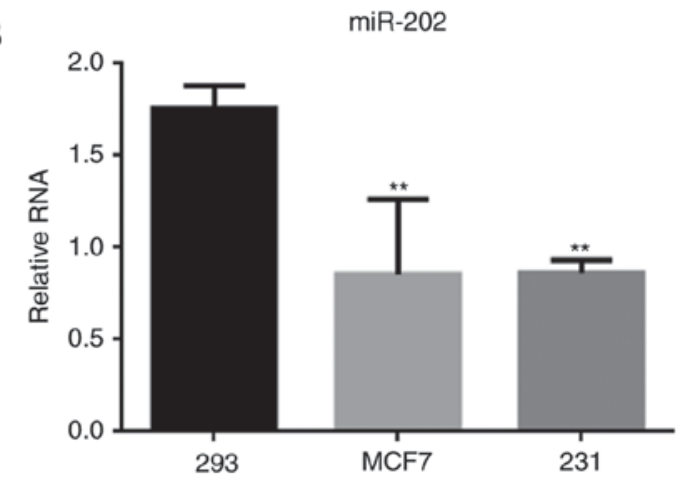

D

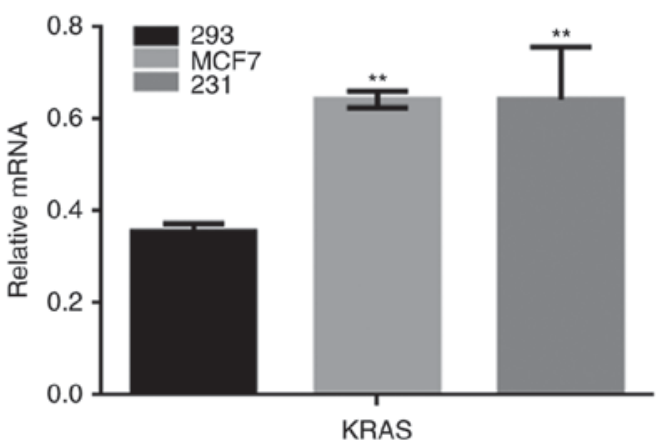

Figure 1. The expression of miR-202 and KRAS in breast cancer. (A) Relative miR-202 expression in 30 pairs of breast cancer tissues and adjacent normal counterpart tissues was detected using real-time PCR. (B) Relative miR-202 expression in 293, MCF7 and MDA-MB-231 cell lines. (C) Relative KRAS expression in 30 pairs of breast cancer tissues and adjacent normal counterpart tissues was detected using real-time PCR. (D) Relative KRAS expression in 293, MCF7 and MDA-MB-231 cell lines. " $\mathrm{P}<0.05$ and ${ }^{* *} \mathrm{P}<0.01$ vs. 293 cells or adjacent normal tissue. miR, micro RNA; PCR, polymerase chain reaction.

tissue. We found that the difference in expression of miR-202 between breast cancer and normal tissues was the largest of the miRNAs identified in our study (Table II). To determine the significance of miR-202 and KRAS expression in breast cancer, the expression of miR-202 was detected using real-time PCR in both breast cancer tissues and in human cell lines, including 293, MCF7 and MDA-MB-231 cells. miR-202 expression was significantly lower in breast cancer tissues than in adjacent tissues (Fig. 1A). We found the expression of miR-202 in MCF7 cells and MDA-MB-231 cells was significantly lower than in normal 293 cells (Fig. 1B). Meanwhile, KRAS expression was obviously up regulated in the 30 breast cancer samples analyzed. Similar results were also observed MCF7 and MDA-MB-231 cells (Fig. 1C, D).

miR-202 inhibits KRAS expression in breast cancer cells. A correlation analysis showed that miR-202 expression was negatively correlated with KRAS expression in breast cancer (Fig. 2A). The miRDB tool indicated that miR-202 could bind to the 3' UTR of KRAS (Fig. 2B). A luciferase reporter assay was used to determine whether KRAS was a potential target gene of miR-202 (Fig. 2C). Co-transfection of KRAS-WT and miR-202 resulted in lower luciferase activity in 293 cells, while co-expression studies with either KRAS-DEL or miR-202 AS (antisense) showed no change in luciferase activity. Further, upon transfection of a miR-202 mimic, downregulation of KRAS expression was observed at both the protein and mRNA levels. Inhibiting miR-202 by transfecting cells with a miR-202 inhibitor resulted in upregulation of KRAS (Fig. 2D-G).
miR-202 inhibits the proliferation of breast cancer cells. An MTT assay was used to examine the effect of miR-202 on cell proliferation. Overexpression of miR-202 significantly blocked the proliferation of both MCF7 and MDA-MB-231 cells (Fig. 3A). By contrast, a lower miR-202 expression level promoted cell proliferation (Fig. 3B).

miR-202 inhibits the metastasis of breast cancer cells. To study the effect of miR-202 on the metastasis of breast cancer cells, transwell assays (with or without Matrigel) were performed. miR-202 significantly suppressed the migration and invasion potential of breast cancer cells (Fig. 4A-D). The above results indicated that miR-202 could inhibit the growth and metastasis of breast cells, possibly by targeting KRAS.

miR-202 inhibits the proliferation and migration of breast cancer cells by targeting KRAS. After generating stable si-KRAS-expressing cells, the cells were transfected with a miR-202 inhibitor or a control inhibitor. The effect of miR-202 inhibition on cell proliferation and migration was detected by MTT and transwell assays (Fig. 5A-C). The results indicated that miR-202 inhibition restored the cell proliferation and migration that were blocked by si-KRAS. Western blot and real time PCR showed that miR-202 inhibition resulted in re-expression of the silenced KRAS (Fig. 5D-E).

The above results indicated that miR-202 could inhibit the growth and metastasis of breast cancer cells by targeting KRAS. 

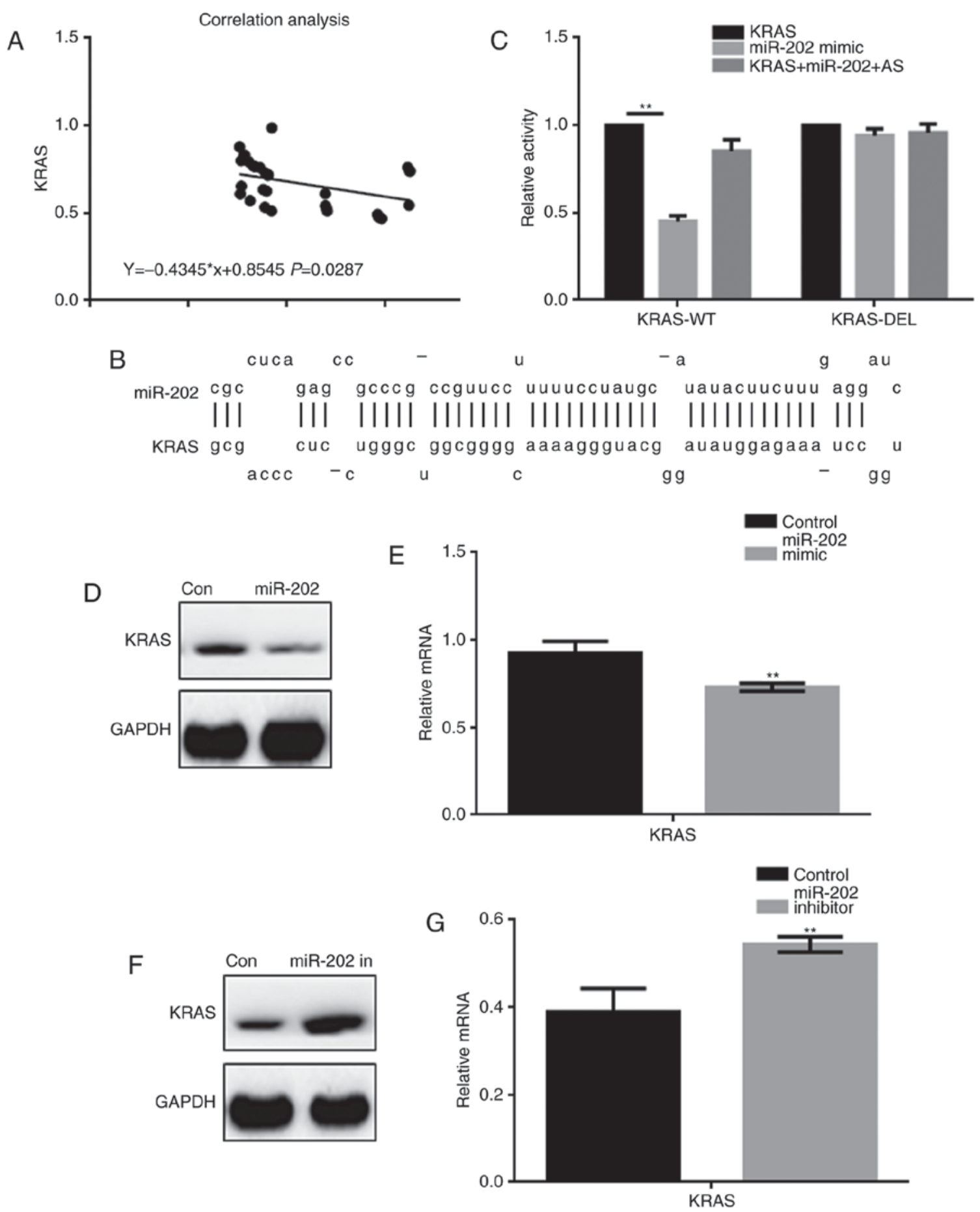

Figure 2. miR-202 inhibits the expression of KRAS in breast cells. (A) Correlation analysis between miR-202 and KRAS expression. The correlation formula is $y=-0.4345 x+0.8545$. (B) The mirDB tool predicted that miR-202 may target KRAS. (C) A luciferase reporter gene assay confirmed that miR-202 was able to target KRAS. (D) The protein and (E) mRNA expression in KRAS was measured in MCF7 cells treated with an miR-202 mimic using western blot and RT-qPCR, respectively. (F) The protein and (G) mRNA expression in KRAS was measured in MCF7 cells treated with an miR-202 inhibitor using western blot and RT-qPCR, respectively. GAPDH was used as an internal loading control. Each bar represents the mean \pm standard deviation of three independent experiments; ${ }^{* *} \mathrm{P}<0.05$ vs. the control. miR, micro RNA; RT-qPCR, reverse transcription-quantitative polymerase chain reaction.

\section{Discussion}

Breast cancer is one of the most common malignant tumors in the world and seriously affects women's physical and mental health. The incidence of breast cancer has been rising since the 1970s (13). Despite marked improvement in the prognosis of breast cancer patients after surgery and adjuvant therapy, breast cancer still has a high rate of recurrence and mortality.
In recent years, with the development of targeted therapies for breast cancer, mortality has declined; however, the mortality rate of breast cancer remains high, and breast cancer is still a major threat to women's health. Therefore, it is important to elucidate the pathogenesis of breast cancer and develop new treatment strategies $(14,15)$.

miRNAs are a group of non-protein coding, short, single-stranded RNAs that are considered to be promising 
A
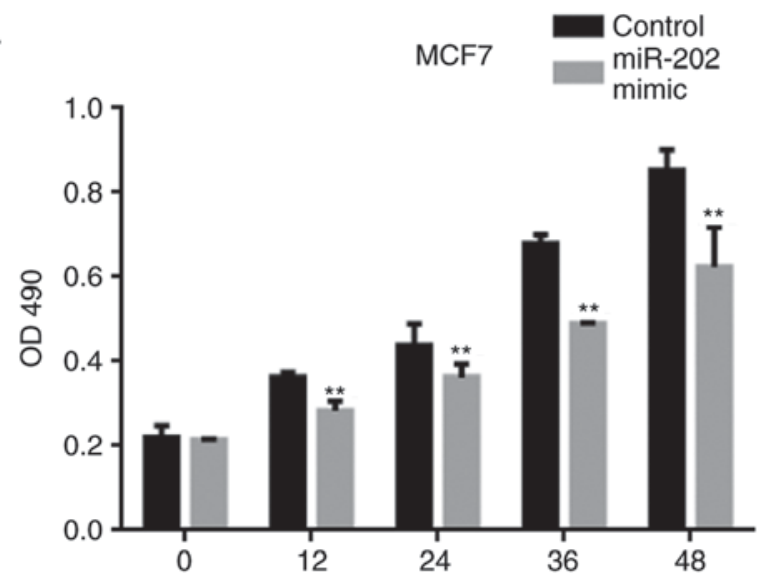

B
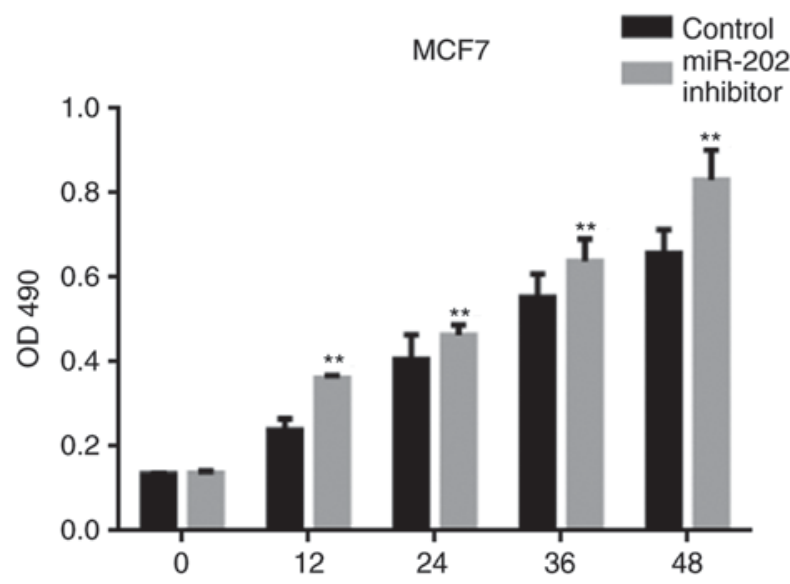
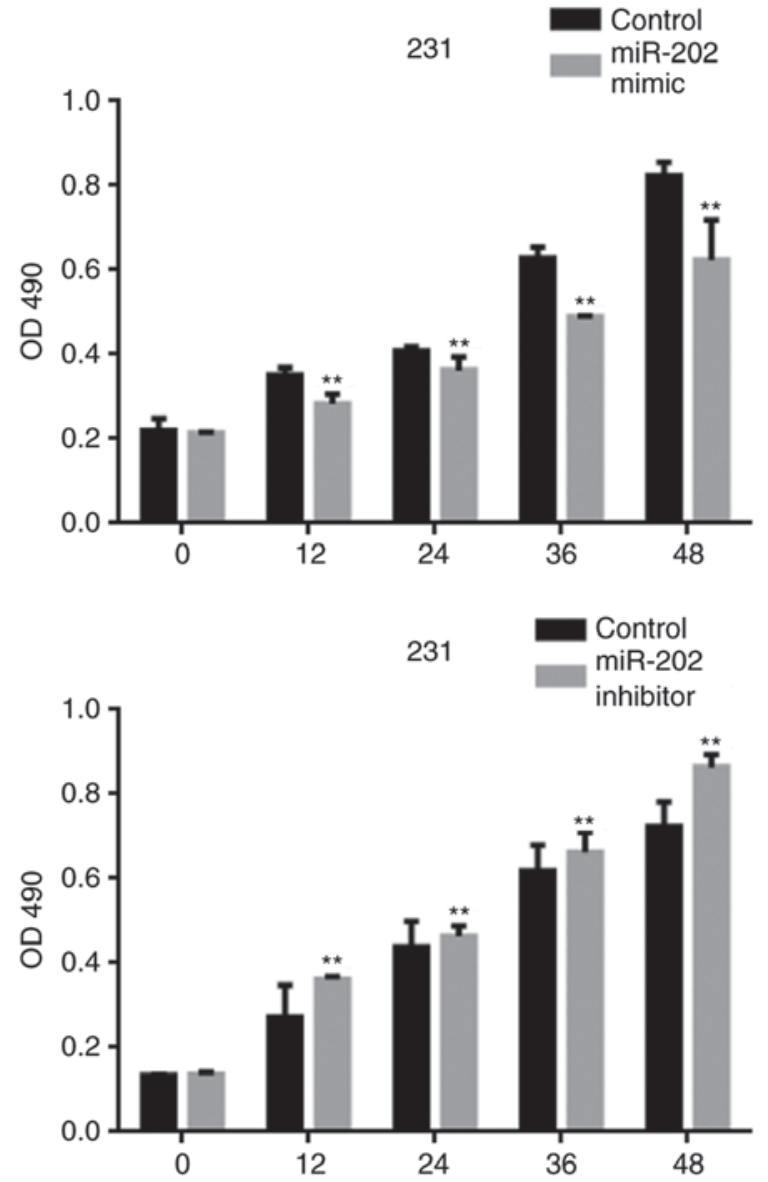

Figure 3. miR-202 inhibits the proliferation of breast cancer cells. (A) Cell proliferation was measured using an MTT assay after 24 h of transfection. MCF7 and MDA-MB-231 cells were transfected with a miR-202 mimic or a scramble control miRNA. (B) Cell proliferation was measured using an MTT assay after $24 \mathrm{~h}$ of transfection. MCF7 and MDA-MB-231 cells were transfected with a miR-202 inhibitor or control miRNA. ${ }^{* *} \mathrm{P}<0.05$ vs. the control. miR, microRNA; OD, optical density.

molecular markers and therapeutic targets in several cancers. miR-202 has been identified as a potential tumor suppressor in various cancers. A previous study revealed that miR-202 upregulation induced novel anticancer effects upregulation in NSCLC and indicated that STAT3 may be a molecular target of miR-202. In human ESCCs, miR-202 regulated apoptosis by targeting HSF2 via mechanism involving caspase-3. A study also identified that miR-202 plays an important role in regulating cell proliferation, migration and invasion of cervical cancer (CC) by directly targeting cyclin D1, and miR-202 may represent a potential therapeutic target for patients with CC (16-22). In addition, a previous study showed that an increased serum concentration of miR-202 may be a strong independent prognostic factor for breast cancer patients (20). Mutations in KRAS lead to sustained activation of the RAS protein, independent of EGFR/HER receptor activation, and eventually cause abnormalities in the RAS signaling pathway. KRAS gene mutations can affect cell growth, proliferation, migration and differentiation and lead to malignant transformation of cells (23-25).

In our study, we found lower expression of miR-202 in breast cancer cells and tissues, indicating that miR-202 may have an inhibitory effect on breast cancer. We identified several potential reasons why miR-202 may have an inhibitory effect on breast cancer. First, we found that miR-202 is negatively correlated with KRAS and can regulate the activity of KRAS to some extent. We also found that overexpression of miR-202 inhibited the proliferation of breast cancer cells. Conversely, when the expression of miR-202 was upregulated, the proliferative capacity of breast cancer cells decreased. Afterwards, we demonstrated that overexpression of miR-202 inhibited the migration and invasion of breast cancer cells. Via a recovery experiment, we also found that miR-202 inhibition could restore cell proliferation and migration blocked by KRAS silencing. In follow-up experiments, the authors intend to further study the effect of miR-202 on breast cancer in vivo.

In summary, our data clarified that miR-202 could inhibit the growth and metastasis of breast cancer cells by targeting KRAS. Although further in vivo tests are needed to confirm our results, our data provide an experimental basis for the treatment of breast cancer.

\section{Acknowledgements}

Not applicable.

\section{Funding}

No funding was received. 
A

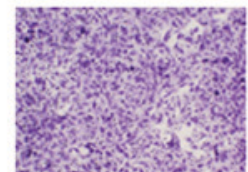

Control

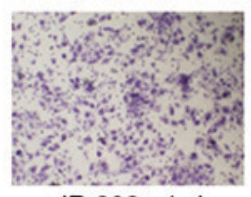

miR-202 mimic

B

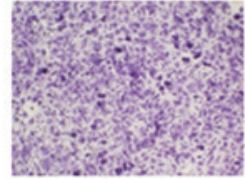

Control

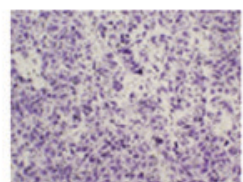

miR-202 inhibitor

C

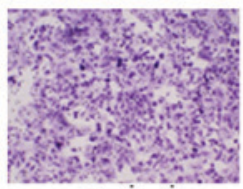

Control

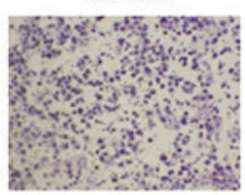

miR-202 mimic

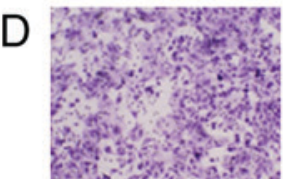

Control

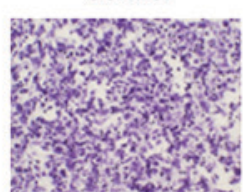

miR-202 inhibitor
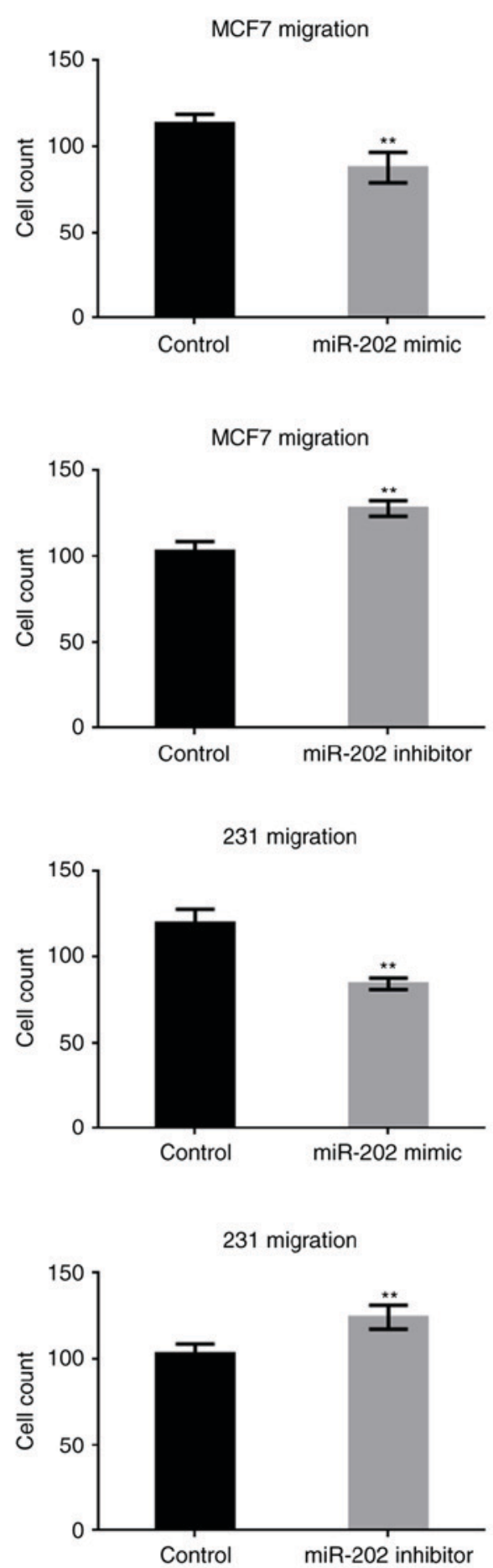
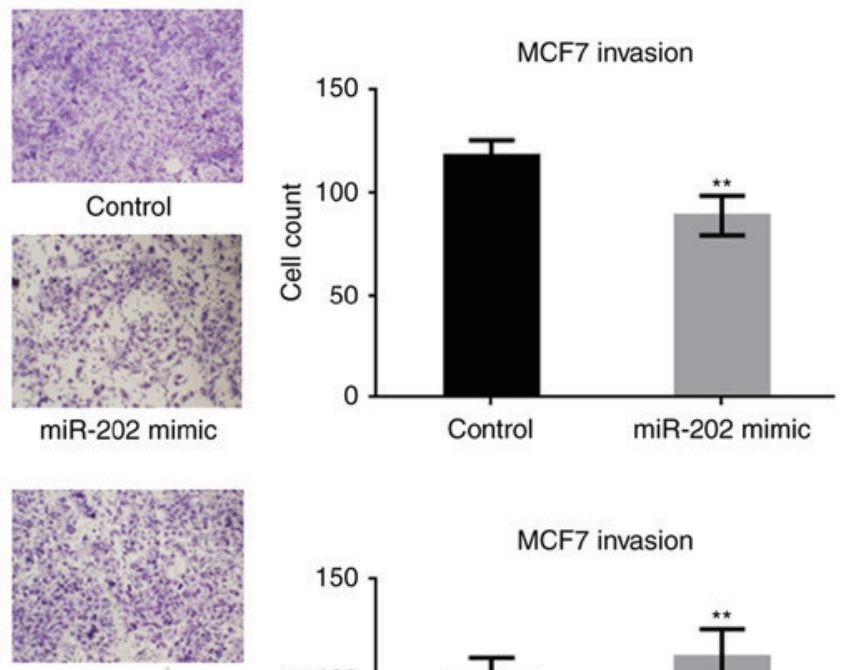

Control

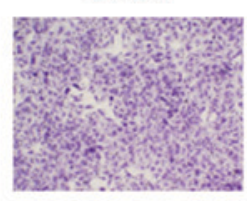

miR-202 inhibitor

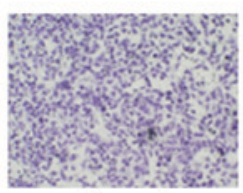

Control

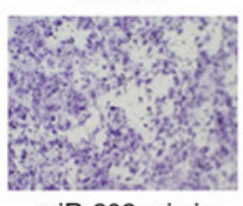

miR-202 mimic
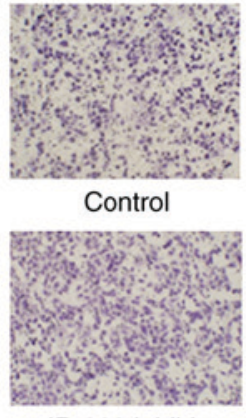

miR-202 inhibitor

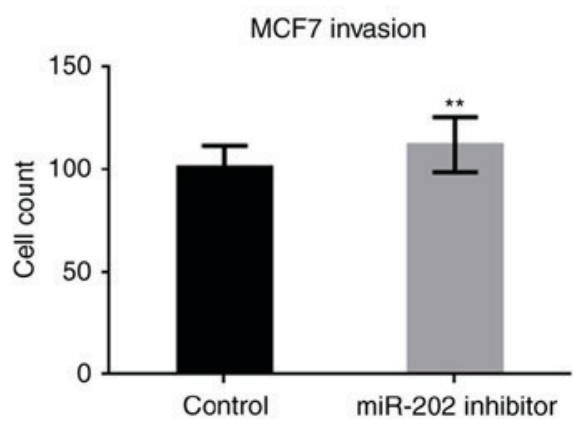

231 invasion
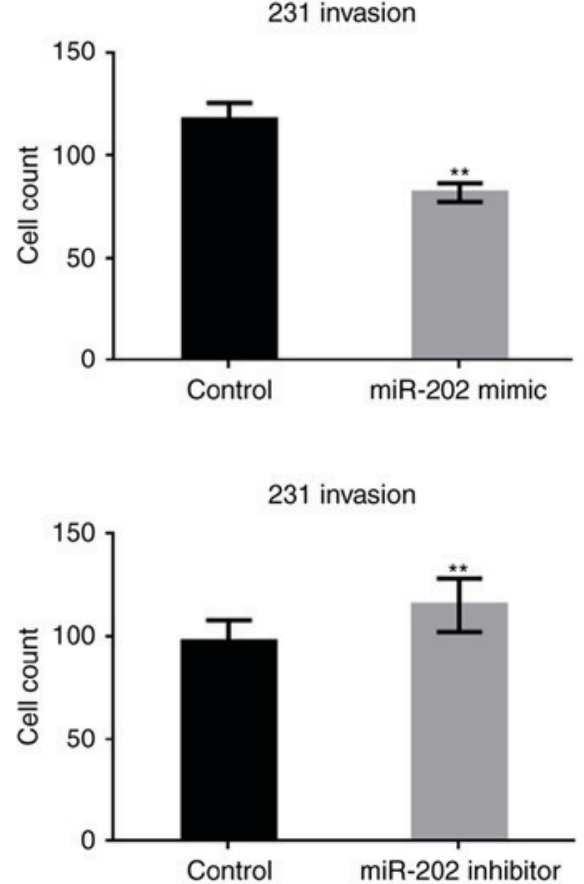

Figure 4. miR-202 inhibits the metastasis of breast cancer cells. (A) The migration capacity of MCF7 cells was measured by a Transwell migration assay after the cells were transfected with a miR-202 mimic or a scramble control miR for $24 \mathrm{~h}$. Overexpression of miR-202 inhibited the migration and invasion of MCF7 cells. (B) The migration capacity of MCF7 cells was measured by a Transwell migration assay after the cells were transfected with a miR-202 inhibitor or scramble control miR for $24 \mathrm{~h}$. Downregulation of miR-202 promoted the migration and invasion of MCF7 cells. (C) The migration capacity of MDA-MB-231 cells was measured by a Transwell migration assay after the cells were transfected with a miR-202 mimic or scramble control miR for 24 h. Overexpression of miR-202 inhibited the migration and invasion of MCF7 cells. (D) The migration capacity of MDA-MB-231 cells was measured by a Transwell migration assay after the cells were transfected with a miR-202 inhibitor or scramble control miR for $24 \mathrm{~h}$. Downregulation of miR-202 promoted the migration and invasion of MCF7 cells. The relative ratio of invasive cells per field is shown. Magnification, $\mathrm{x} 400$. ${ }^{* *} \mathrm{P}<0.05$, vs. the control. miR, microRNA.

\section{Availability of data and materials}

The datasets used and/or analyzed during the current study are available from the corresponding author on reasonable request.

\section{Authors' contributions}

JT conceived and designed the study, acquired the specimens and data, directed the drafting of manuscript and revised it, 
A

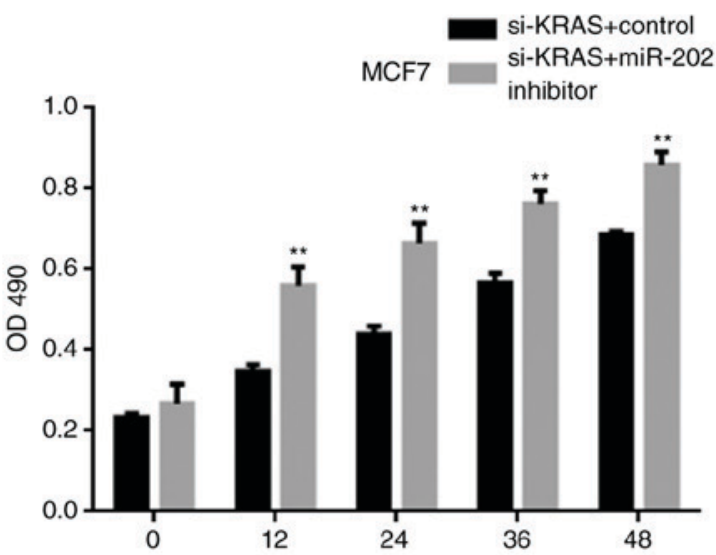

B

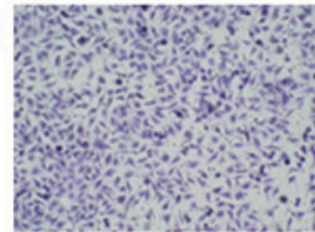

si-KRAS
si-KRAS+miR-202 in

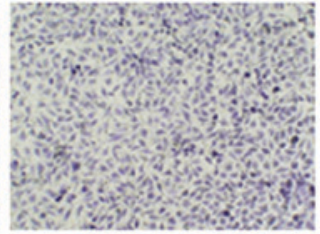

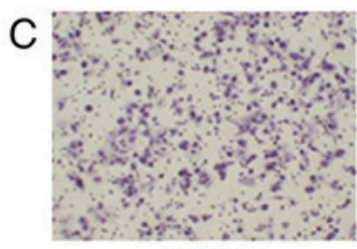

Si-KRAS

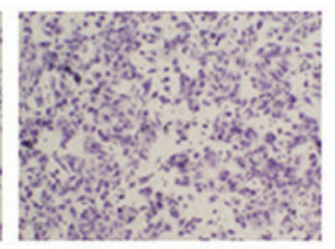

si-KRAS+miR-202 in
D Si-KRAS si-KRAS+

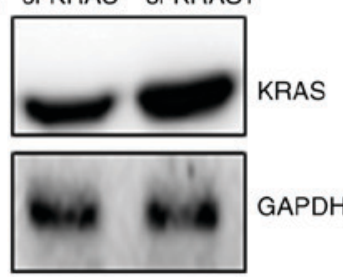

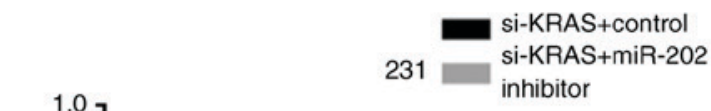

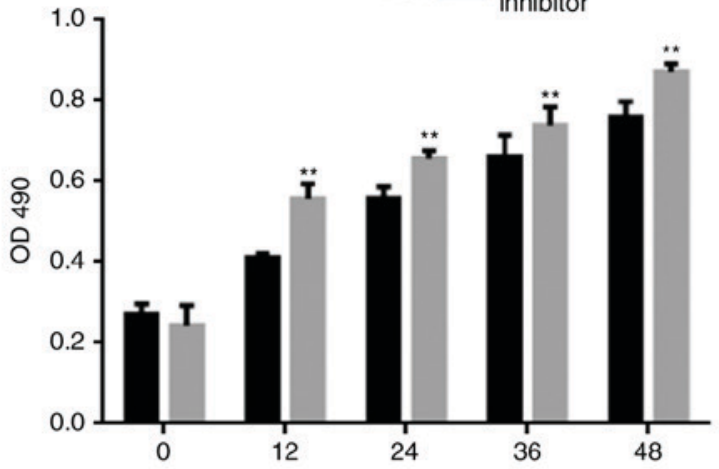

MCF7 migration
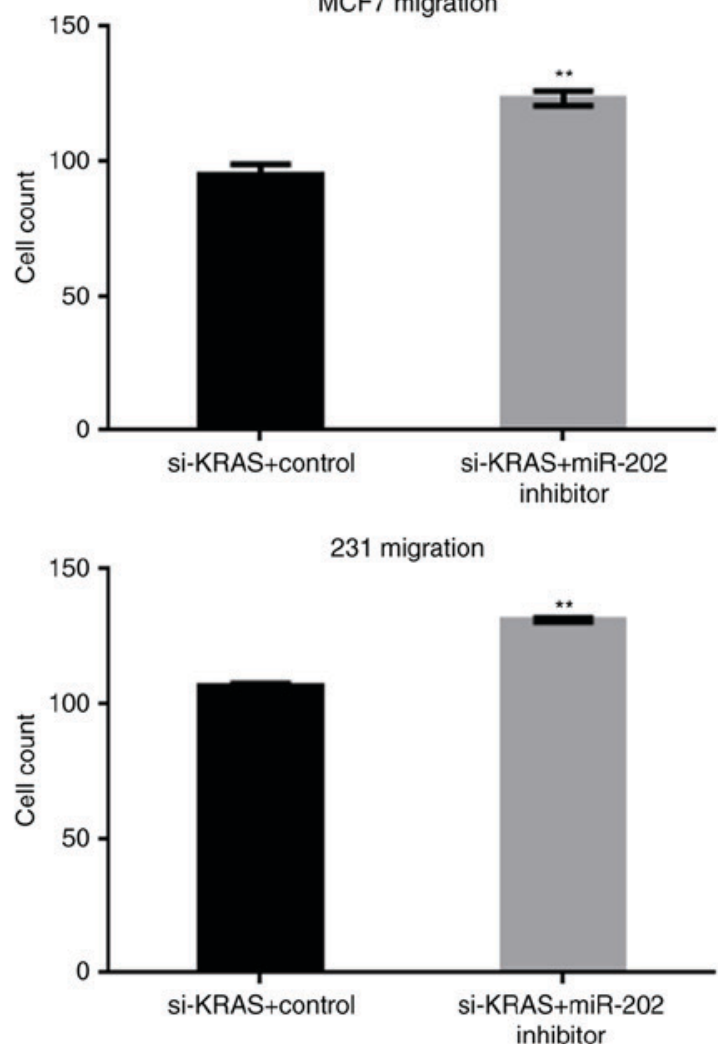

E

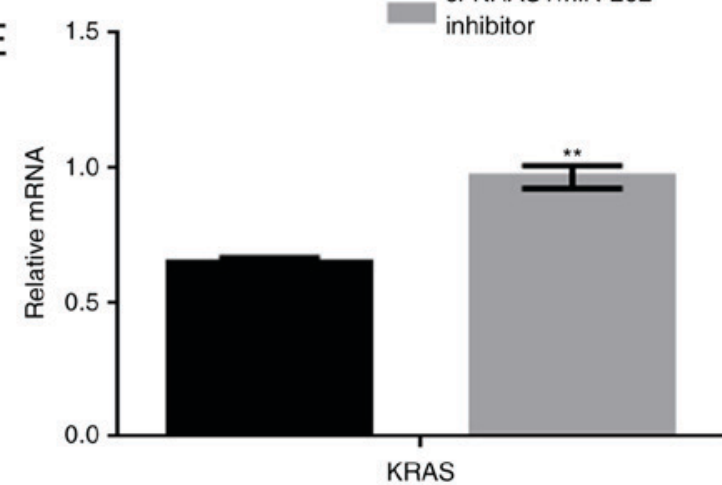

Figure 5. miR-202 inhibits the proliferation and migration of breast cancer cells by targeting KRAS. (A) Cell proliferation was measured using an MTT assay after $24 \mathrm{~h}$ of transfection. After transfection with si-KRAS, MCF7 and MDA-MB-231 cells were transfected with a miR-202 inhibitor or control miR. Cell migration was measured using a Transwell assay after $24 \mathrm{~h}$ of transfection. After transfection with si-KRAS, (B) MCF7 and (C) MDA-MB-231 cells were transfected with a miR-202 inhibitor or control miR. After transfection with si-KRAS MCF7 cells were transfected with a miR-202 inhibitor or control miR. Magnification, $x 400$. The (D) protein and (E) mRNA levels of KRAS were measured in MCF7 cells using western blot and reverse transcription-quantitative polymerase chain reaction, respectively. GAPDH was used as an internal loading control. Each bar represents the mean \pm standard deviation of three independent experiments. ${ }^{* *} \mathrm{P}<0.05$, vs. the control. Si, small interfering; miR, microRNA. 
and provided the financial support. SG designed the study and took responsibility for the experiments. CC performed the metastasis assay, QD performed the statistical analysis. JC performed the western blot analysis.

\section{Ethics approval and consent to participate}

All patients provided written informed consent. The project was approved by the Ethics Committee for Medical Science Research of Zhongnan Hospital of Wuhan University.

\section{Consent for publication}

All patients provided written informed consent for the publication of their data.

\section{Competing interests}

The authors confirm that they have no competing interests.

\section{References}

1. Rizzo S, Cangemi A, Galvano A, Fanale D, Buscemi S, Ciaccio M, Russo A, Castorina S and Bazan V: Analysis of miRNA expression profile induced by short term starvation in breast cancer cells treated with doxorubicin. Oncotarget 8: 71924-71932, 2017.

2. Mandujano-Tinoco EA, Garcia-Venzor A, Muñoz-Galindo L, Lizarraga-Sanchez F, Favela-Orozco A, Chavez-Gutierrez E, Krötzsch E, Salgado RM, Melendez-Zajgla J and Maldonado V: miRNA expression profile in multicellular breast cancer spheroids. Biochim Biophys Acta 1864: 1642-1655, 2017.

3. Li P, Xu T, Zhou X, Liao L, Pang G, Luo W, Han L, Zhang J, Luo X, Xie X and Zhu K: Downregulation of miRNA-141 in breast cancer cells is associated with cell migration and invasion: Involvement of ANP32E targeting. Cancer Med 6: 662-672, 2017.

4. Lykov AP, Kabakov AV, Kazakov OV, Bondarenko NA, Poveshchenko OV, Raiter TV, Poveshchenko AF, Strunkin DN and Konenkov VI: Levels of miRNA and hormones in thoracic duct lymph in rats with experimental breast cancer induced by N-methyl-N-nitrosourea. Bull Exp Biol Med 162: 387-390, 2017.

5. Bhardwaj A, Singh H, Rajapakshe K, Tachibana K, Ganesan N, Pan Y, Gunaratne PH, Coarfa C and Bedrosian I: Regulation of miRNA-29c and its downstream pathways in preneoplastic progression of triple-negative breast cancer. Oncotarget 8 : 19645-19660, 2017.

6. Danza K, Summa S, Pinto R, Pilato B, Palumbo O, Carella M, Popescu O, Digennaro M, Lacalamita R and Tommasi S: TGFbeta and miRNA regulation in familial and sporadic breast cancer. Oncotarget 8: 50715-50723, 2017.

7. Fang H, Xie J, Zhang M, Zhao Z, Wan Y and Yao Y: miRNA-21 promotes proliferation and invasion of triple-negative breast cancer cells through targeting PTEN. Am J Transl Res 9: 953-961, 2017.

8. Zhang L, Xu J, Yang G, Li H and Guo X: miR-202 inhibits cell proliferation, migration, and invasion by targeting EGFR in human bladder cancer. Oncol Res, Jan 3, 2018 (Epub ahead of print).
9. Kim RK, Suh Y, Yoo KC, Cui YH, Kim H, Kim MJ, Gyu Kim I and Lee SJ: Activation of KRAS promotes the mesenchymal features of basal-type breast cancer. Exp Mol Med 47: e137, 2015.

10. Kim Y, Kim J, Lee HD, Jeong J, Lee W and Lee KA: Spectrum of EGFR gene copy number changes and KRAS gene mutation status in Korean triple negative breast cancer patients. PLoS One 8: e79014, 2013.

11. McVeigh TP, Jung SY, Kerin MJ, Salzman DW, Nallur S, Nemec AA, Dookwah M, Sadofsky J, Paranjape T, Kelly O, et al: Estrogen withdrawal, increased breast cancer risk and the KRAS-variant. Cell Cycle 14: 2091-2099, 2015.

12. Su X, Zhang L, Li H, Cheng P, Zhu Y, Liu Z, Zhao Y, Xu H, Li D, Gao $\mathrm{H}$ and Zhang T: MicroRNA-134 targets KRAS to suppress breast cancer cell proliferation, migration and invasion. Oncol Lett 13: 1932-1938, 2017.

13. Zeng Y, Zhou Z, Fan M, Gong T, Zhang Z and Sun X: PEGylated cationic vectors containing a protease-sensitive peptide as a miRNA delivery system for treating breast cancer. Mol Pharm 14: 81-92, 2017.

14. Zhang J, Le TD, Liu L and Li J: Inferring miRNA sponge co-regulation of protein-protein interactions in human breast cancer. BMC Bioinformatics 18: 243, 2017.

15. Zhang L, Yang X, Lv Y, Xin X, Qin C, Han X, Yang L, He W and Yin L: Cytosolic co-delivery of miRNA-34a and docetaxel with core-shell nanocarriers via caveolae-mediated pathway for the treatment of metastatic breast cancer. Sci Rep 7: 46186, 2017.

16. Zhao Z, Lv B, Zhang L, Zhao N and Lv Y: miR-202 functions as a tumor suppressor in non-small cell lung cancer by targeting STAT3. Mol Med Rep 16: 2281-2289, 2017.

17. Mody HR, Hung SW, Pathak RK, Griffin J, Cruz-Monserrate Z and Govindarajan R: miR-202 diminishes TGF $\beta$ receptors and attenuates TGF $\beta 1$-Induced EMT in pancreatic cancer. Mol Cancer Res 15: 1029-1039, 2017.

18. Zhang Y, Chen Z, Li MJ, Guo HY and Jing NC: Long non-coding RNA metastasis-associated lung adenocarcinoma transcript 1 regulates the expression of Gli2 by miR-202 to strengthen gastric cancer progression. Biomed Pharmacother 85: 264-271, 2017.

19. Yi Y, Li H, Lv Q, Wu K, Zhang W, Zhang J, Zhu D, Liu Q and Zhang W: miR-202 inhibits the progression of human cervical cancer through inhibition of cyclin D1. Oncotarget 7: 72067-72075, 2016.

20. Farhana L, Dawson MI and Fontana JA: Down regulation of miR-202 modulates Mxd1 and Sin3A repressor complexes to induce apoptosis of pancreatic cancer cells. Cancer Biol Ther 16: 115-124, 2016

21. Joosse SA, Müller V, Steinbach B,Pantel K and Schwarzenbach H: Circulating cell-free cancer-testis MAGE-A RNA, BORIS RNA, let-7b and miR-202 in the blood of patients with breast cancer and benign breast diseases. Br J Cancer 111: 909-917, 2014.

22. Zhao Y, Li C, Wang M, Su L, Qu Y, Li J, Yu B, Yan M, Yu Y, Liu B and Zhu Z: Decrease of miR-202-3p expression, a novel tumor suppressor, in gastric cancer. PLoS One 8: e69756, 2013.

23. Cejalvo JM, Pérez-Fidalgo JA, Ribas G, Burgués O, Mongort C, Alonso E, Ibarrola-Villava M, Bermejo B, Martínez MT, Cervantes A and Lluch A: Clinical implications of routine genomic mutation sequencing in PIK3CA/AKT1 and KRAS/NRAS/BRAF in metastatic breast cancer. Breast Cancer Res Treat 160: 69-77, 2016.

24. Ustinova M, Daneberga Z, Bērziña D, Nakazawa-Miklaševiča M, Maksimenko J, Gardovskis J and Miklaševičs E: Impact of KRAS variant rs61764370 on breast cancer morbidity. Exp Oncol 37: 292-294, 2015.

25. Huang X, Yang Y, Guo Y, Cao ZL, Cui ZW, Hu TC and Gao LB: Association of a let-7 KRAS rs712 polymorphism with the risk of breast cancer. Genet Mol Res 14: 16913-16920, 2015. 